\title{
Mini-mental state examination in neurological patients
}

\author{
JPR DICK, RJ GUILOFF, ${ }^{*}$ A STEWART, J BLACKSTOCK, C BIELAWSKA, EA PAUL, $\dagger$ \\ CD MARSDEN
}

From the University Department of Neurology, Institute of Psychiatry and King's College Hospital, London, $U K$

SUMMARY The Mini-Mental State examination has been found to be a quick and valuable test for simple bedside screening, and for serial assessment of cognitive function in a population of 126 neurological patients. Amongst those with cognitive impairment, there was a close relation between the Mini-Mental State examination and the conventional Weschler Adult Intelligence Scale (WAIS). However, the Mini-Mental test was not a sensitive indicator of focal versus diffuse hemisphere disease. Further refinement in the areas of language and visuo-spatial function may improve its value.

Assessment of cognitive function is essential for accurate diagnosis and management in general neurological practice. Detailed assessment by the neurologist and psychologist requires a high degree of specialist training and is time-consuming. It is desirable to have a standardised simple and quick test of cognitive function which could be used routinely by the admitting physician. The MiniMental State examination (MMS) was designed to detect patients with cognitive impairment amongst a psychiatric population. ${ }^{1}$ While it does not evaluate the form of thinking or the presence of abnormal mental experiences or mood, it was described as thorough in the cognitive realm. It is quick (it takes 5 minutes, as against the WAIS which takes $60-90$ minutes to administer), and it is easy to undertake. We have assessed the usefulness of the MMS examination in a neurological population.

\section{Materials and methods}

One-hundred-and-twenty-six consecutive patients (mean age $49 \cdot 9$ years, standard deviation (SD) $17 \cdot 4$, range $13-$ 83 ), of whom 61 were male (mean age $47 \cdot 5$, SD 18.0), and 65 were female (mean age $52 \cdot 1$ years, SD $16 \cdot 5$ ) were

\footnotetext{
${ }^{*}$ Present address and address for reprint requests: RJ Guiloff, Westminster Hospital, Dean Ryle Street, London SW1 2AP, UK.

†Computing and Statistics Advisory Unit, Institute of Neurology, Queen Square, London WC1N 3BG, UK.
}

Received 6 May 1983 and in final revised form 26 October 1983. Accepted 18 November 1983 examined on admission to acute neurological and neurosurgical wards. There were 115 right-handers, seven left-handers and four for whom handedness was unknown.

A diagnosis was established with the aid of appropriate investigations and surgery when indicated; investigations included computed tomography (CT scan), carotid angiography, myelography, electrophysiological studies and relevant haemotological, biochemical and bacteriological tests.

On the basis of all clinical and laboratory data, the 126 patients were classified into nine groups according to the anatomical site(s) of their lesion(s). There were 45 patients with bilateral hemisphere disease, 16 patients with left hemisphere disease, 14 patients with right hemisphere disease, 14 patients with brain stem disease, 17 patients with spinal cord disease, six patients with peripheral nerve disease, two patients with muscle disease, six patients with other neurological diseases, and six who had no neurological disease. The 31 patients with disease of the spinal cord, peripheral nerve and muscle, or other neurological diseases have been used as neurological controls. No difference in mean age and sex ratio between the controls and either the whole patient population, or any individual group was apparent. Those with brain stem disease were not analysed separately, for some exhibited hydrocephalus. All 30 of the patients with unilateral hemisphere disease had CT scans; in addition, seven had carotid angiography and 10 required surgery. Three patients with left hemisphere disease and one patient with right hemisphere disease were excluded from inter-group analysis, because they were either left-handed or their handedness was unknown. Forty-three of the 45 patients with bilateral hemisphere disease had CT scans.

Patients were further categorised into those who were mentally intact $(n=93,45$ male and 48 female, mean age 48.5 years, SD 16.7 ), and those who, on bedside neurolog- 
ical examination by three neurologists, were diagnosed as having cognitive impairment $(n=33,16$ male and 17 female, mean age 54.0 years, SD 18.8). This latter category included those who showed evidence of mild or gross generalised intellectual deficit, or mild or gross focal cortical dysfunction (aphasia, acalculia, agraphia, etc). Subsequent to the initial study of the consecutive series of 126 patients, we studied a further 17 patients, selected because they exhibited cognitive impairment, to give a total of 50 patients in this category for further analysis.

The MMS was administered to all patients on admission by the duty neurological senior house physician. The original MMS test was slightly adapted for use in the United Kingdom, by replacing the request to name the state, county, town or city, hospital, and floor with the name of the ward, hospital, district, town and country (Appendix). The test was repeated 24 hours later on 45 patients, in 30 by the same observer and in 15 by a different observer. Fourteen clinically stable patients were retested after a longer period (mean interval 31 days, range 7-70) by their initial observer. Four patients were followed with repeated MMS tests during the course of their illness. Twenty patients in the original series of 126 , and the additional 17 cognitively impaired patients, were tested by trained psychologists using the Weschler Adult Intelligence Scale. ${ }^{2}$ The WAIS IQ scores were used for subsequent analysis.

The MMS scores were found to have a skewed distribution and non-parametric statistics were therefore used to analyse these data.

\section{Results (Table 1-3)}

\section{RELIABILITY OF THE MMS SCORE}

Using the Wilcoxon Matched-pairs signed rank test, there was no difference between the two total MMS scores of the 15 cases tested by different observers at an interval of 24 hours. This was also true for the 30 cases tested by the same observer at a 24 hour interval, and for the 14 clinically stable patients tested by the same observer at a mean interval of 31 days (range 7-70 days). For comparison with the test-retest reliability obtained by Folstein et al the Pearson Correlation coefficient, which those authors employed, was computed. Pearson Correlation coefficients were close to those quoted by Folstein ( 0.92 for patients retested by the same observer and 0.95 for patients retested by a different observer). The Kendall Correlation coefficients (a non-parametric measure more appropriate for data
Table 1 Sensitivity and specificity of the MMS examination

\begin{tabular}{llccc}
\hline & $\begin{array}{l}\text { With Cognitive } \\
\text { Impairment } \\
(n=50)\end{array}$ & \multicolumn{2}{l}{$\begin{array}{l}\text { Without Cognitive } \\
\text { Impairment } \\
(n=93)\end{array}$} \\
\hline MMS total score & $\mathrm{n}$ & $\%$ & $\mathrm{n}$ & $\%$ \\
$0-20$ & 28 & 56 & 0 & 0 \\
$0-21$ & 28 & 56 & 0 & 0 \\
$0-22$ & 34 & 68 & 1 & $1 \cdot 1$ \\
$0-23$ & 38 & 76 & 4 & $4 \cdot 3$ \\
$0-24$ & 42 & 84 & 7 & $7 \cdot 5$ \\
$0-25$ & 45 & 90 & 13 & 14 \\
$0-26$ & 47 & 94 & 17 & $18 \cdot 4$ \\
$0-27$ & 50 & 100 & 25 & $27 \cdot 7$ \\
$0-28$ & 50 & 100 & 36 & $38 \cdot 8$ \\
$0-29$ & 50 & 100 & 67 & $79 \cdot 1$ \\
$0-30$ & 50 & 100 & 93 & 100 \\
\hline
\end{tabular}

$\mathbf{n}=$ number of patients with a given MMS score in the group of those with (total 50) and those without (total 93) cognitive impairment.

A MMS score of 23 or less gave $4.3 \%$ false positive results in the cognitively normal group, and detected $76 \%$ of the cognitively impaired.

Table 3 MMS scores by diagnostic group pair-wise comparison using the Mann-Whitney U-test

\begin{tabular}{llll}
\hline & Controls & $\begin{array}{l}\text { Left } \\
\text { Hemisphere }\end{array}$ & Bilateral \\
\hline Right hemisphere & $\mathbf{u}=127$ & $\mathrm{u}=128$ & $\mathrm{u}=102$ \\
& $\mathrm{p}=0.20$ & $\mathrm{p}=0.0152$ & $\mathrm{p}=0.0025$ \\
Controls & & $\mathrm{u}=312$ & $\mathrm{u}=979$ \\
Left hemisphere & & $\mathrm{p}=0.057$ & $\mathrm{p}=0.003$ \\
& & & $\mathrm{u}=329$ \\
& & $\mathrm{p}=0.88$ \\
\hline
\end{tabular}

that is not normally distributed) were 0.65 for the group retested by the same observer and 0.63 for the group retested by different observers.

\section{CAPACITY OF THE MMS SCORE TO DETECT COGNITIVE IMPAIRMENT}

The Mann-Whitney $U$ test showed that the 33 patients in the initial series of 126 patients with cognitive impairment, judged by clinical bedside examination, had lower MMS scores than those with normal cognitive function $(U=145, p=0 \cdot 0001)$. There was a relationship (Spearman correlation coefficient) between the WAIS IQ scores obtained in the 20 patients so studied and the MMS total score (verbal IQ, $r_{s}=0.55, p=0.01$; performance

Table 2 Mean total test scores in subgroups of 126 neurological patients

\begin{tabular}{|c|c|c|c|c|c|c|c|c|c|}
\hline & $n$ & $M$ & $F$ & $\begin{array}{l}\text { Mean Age } \\
\text { (years) }\end{array}$ & $\begin{array}{l}S D \\
\text { (years) }\end{array}$ & $\begin{array}{l}\text { Cognitively } \\
\text { Impaired }\end{array}$ & $\begin{array}{l}\text { Mean Total } \\
\text { MMS scores }\end{array}$ & $S D$ & Range \\
\hline $\begin{array}{l}\text { Neurological controls } \\
\text { Bilateral hemisphere } \\
\text { Right hemisphere } \\
\text { Left hemisphere }\end{array}$ & $\begin{array}{l}31 \\
45 \\
14 \\
16\end{array}$ & $\begin{array}{r}11 \\
27 \\
8 \\
8\end{array}$ & $\begin{array}{r}20 \\
18 \\
6 \\
8\end{array}$ & $\begin{array}{l}47 \cdot 0 \\
48 \cdot 3 \\
56 \cdot 5 \\
57 \cdot 7\end{array}$ & $\begin{array}{l}13 \cdot 3 \\
19 \cdot 3 \\
15 \cdot 6 \\
16 \cdot 4\end{array}$ & $\begin{array}{r}0 \\
21 \\
2 \\
7\end{array}$ & $\begin{array}{l}27 \cdot 7 \\
23 \cdot 4 \\
28 \cdot 3 \\
23 \cdot 1\end{array}$ & $\begin{array}{l}2 \cdot 4 \\
6 \cdot 8 \\
1 \cdot 8 \\
7 \cdot 2\end{array}$ & $\begin{array}{r}23-30 \\
0-30 \\
24-30 \\
8-30\end{array}$ \\
\hline
\end{tabular}


IQ, $r_{\mathrm{s}}=0.56, \mathrm{p}=0.02$, and total WAIS IQ, $\mathrm{r}_{\mathrm{s}}=$ $0.52, p=0.02$ ). As only 13 of these 20 patients were cognitively impaired on clinical testing, we checked that the relationship was significant after including 17 additional patients with cognitive impairment. The Spearman correlation coefficients between WAIS IQs and MMS total scores in these 30 patients were, for verbal IQ, $r_{s}=0.45(p=$ $0.02)$; for performance IQ, $r_{s}=0.58(p=0.004)$, and for total WAIS IQ, $r_{s}=0.54(p=0.0006)$.

\section{SENSITIVITY AND SPECIFICITY OF THE MMS \\ EXAMINATION TO DETECT COGNITIVE \\ IMPAIRMENT}

The capacity of the MMS examination to detect cognitive impairment was assessed by examination of the capacity of a range of MMS score "cut-off" points (table 1) to pick up the abnormality evident clinically in those 50 patients judged to have cognitive impairment. The capacity of the MMS examination to produce false positive abnormal low scores amongst those judged clinically to be cognitively normal was also assessed in the 93 patients who were thought to show no evidence of such abnormalities (table 1).

\section{MMS SCORES IN FOCAL OR GENERALISED BRAIN} DISEASE

Kruskal-Wallis one-way analysis of variance showed that there were differences in MMS scores in different diagnostic groups (table 2$)\left(\chi^{2}=17 \cdot 05, p=\right.$ $0 \cdot 04)$. MMS scores in the important diagnostic groups taken in pairs were then compared using the Mann-Whitney U test (table 3).

The MMS total scores in those with right hemisphere disease did not differ from the control group, but they were greater than those in the left hemisphere and bilateral hemisphere groups. The MMS total scores in the left hemisphere group did not differ from the bilateral hemisphere group. The MMS total scores of those with left hemisphere disease was lower than those of the control group. This difference nearly reached statistical significance $(p$ $=0.057)$. The MMS total scores in the bilateral hemisphere group were lower than those of the control group.

\section{Discussion}

We have concluded that the MMS examination produces reliable scores which do not appear to be influenced by repetition or learning, and that consistent results are obtained by the same or a different observer on repeat testing after 24 hours. Folstein $e t$ $a l^{1}$ reported similar results amongst a psychogeriatric population.
All patients classified clinically as having no cognitive impairment in our study had total MMS scores of more than 21. Folstein et al ${ }^{1}$ used an MMS total score of less than 20 to separate patients with dementia or functional psychosis from normal subjects and those with anxiety neurosis or personality disorder. They reported in that study that all of 63 elderly retired residents assumed to be intellectually intact scored 24 points or more. In their study of a neurological population, De Paulo et $a^{3}$ used a MMS total score of 23 or less to separate those with cognitive impairment from those without.

Four of our population of 93 neurological patients with intact cognition had a total MMS score of 23 or less; they were notable for low educational achievement. Lowering the cut-off point to 22 or 21 avoided misclassification of normal individuals as cognitively impaired. However, reducing the cut-off point decreased the sensitivity (that is increased the number of false negatives). Some of those 50 patients classed as cognitively impaired on clinical grounds had total MMS scores as high as 27 , and 12 of them had MMS scores of 24 or more (table 1). Optimum specificity and sensitivity were achieved by regarding a score of 23 or less as suggestive of cognitive impairment. However, it must be emphasised that some cognitively impaired subjects scored more than 23 , and some cognitively intact patients scored less than 23. The MMS therefore is not an entirely reliable indicator of cognitive function and must be interpreted in the light of all other clinical data.

Nevertheless, overall, there was good general agreement between WAIS IQs and MMS total scores; similar results also were found in a psychogeriatric population ${ }^{1}$ and in a neurological population. ${ }^{3}$

The MMS test did not seem useful in differentiating focal from "diffuse" brain disease. Patients in the left hemisphere and bilateral hemisphere group behaved similarly in the MMS test, while patients in the right hemisphere group behaved similarly to the control group and differently from those in the left hemisphere and bilateral hemisphere groups. Clearly there is a verbal bias in the administration of the MMS test, and despite the fact that the MMS total scores showed a correlation with both performance and verbal IQs, it was relatively insensitive to damage of the right hemisphere. Further refinement of the test to include greater exploration of visuospatial function might improve its value. In addition, the MMS does not investigate language function in detail. Mayeux et $\mathrm{al}^{4}$ have added a number of items to the original MMS in order to examine language functions in more detail.

We conclude that in neurological patients the 
MMS examination was a valuable, consistent and rapid method for routine bedside assessment of the cognitive state, and for serial assessment of cognitive deficit. However, it was not a sensitive indicator of focal versus diffuse hemisphere disease and in particular of left versus bilateral hemisphere disease. Further refinement of the test to expand on examination of language and visuo-spatial functions may enhance its value as a routine instrument in neurological practice.

We are grateful to Dr Brenda Gardiner for helpful criticism.

\section{Appendix}

\section{THE MINI-MENTAL STATE EXAMINATION}

Orientation

Score one point for correct answers to each of the following questions:

What is the time?

$$
\begin{aligned}
& \text { date? } \\
& \text { day? } \\
& \text { month? }
\end{aligned}
$$$$
\text { year? }
$$

5 points $(\quad)$

What is the name of this ward?

$$
\text { the hospital? }
$$

the district?

the town?

and the country?

the country? 5 points $(\quad)$

\section{Registration}

Name three objects. Score up to 3 points if, at the first attempt, the patient repeats, in order, the 3 objects you have randomly named. Score 2 or one if this is the number of objects he repeats correctly. Endeavour, by further attempts and prompting, to have all 3 repeated, so as to test recall later.

$$
3 \text { points }(\quad)
$$

\section{Attention and calculation}

Ask the patient to subtract 7 from 100, and then 7 from the result-repeat this 5 times, scoring one for each time a correct subtraction is performed.
Recall

Ask for the 3 objects repeated in the registration test, scoring one for each correctly recalled.

$$
3 \text { points }(\quad)
$$

\section{Language}

Score one point for 2 objects (a pencil and a watch) correctly named.

$$
2 \text { points ( ) }
$$

Score one point if the following sentence is correctly repeated. "No ifs, ands or buts".

$$
1 \text { point ( ) }
$$

Score 3 if a 3-stage command is correctly executed, score one for each stage; for example, "with the index finger of your right hand touch the tip of your nose and then your left ear", or "take this piece of paper in your right hand, fold it in half and place in on the floor".

3 points ( )

On a blank piece of paper write, "Close your eyes" and ask the patient to obey what is written. Score one point if he closes his eyes.

1 point ( )

Ask the patient to write a sentence. Score one if the sentence is sensible and has a verb and a subject.

1 point ( )

Construct a pair of intersecting pentagons, each side one inch long. Score one if this is correctly copied.

1 point ( )

TOTAL SCORE $(=30)(\quad)$

\section{References}

' Folstein MF, Folstein SE, McHugh PR. "Mini-Mental State" A Practical Method for Grading the Cognitive State of patients for the Clinician. J Psychiat Res 1975;12:189-98.

${ }^{2}$ Wechsler D. Weschler Adult Intelligence Scale. Psychol. Corp. New York, 1955.

${ }^{3}$ DePaulo JR, Folstein MF, Gordon B. Psychiatric screening on a neurological ward. Psychol Med 1980;10: 125-32.

${ }^{4}$ Mayeux R, Stern Y, Rosen J, Leventhal J. Depression, intellectual impairment and Parkinson's disease. Neurology (NY) 1981;31:645-50. 